\title{
Voluntary Control over Prestimulus Activity Related to Encoding
}

\author{
Matthias J. Gruber and Leun J. Otten \\ Institute of Cognitive Neuroscience, University College London, London, WC1N 3AR, United Kingdom
}

A new development in our understanding of human long-term memory is that effective memory formation relies on neural activity just before an event. It is unknown whether such prestimulus activity is under voluntary control or a reflection of random fluctuations over time. In the present study, we addressed two issues: (1) whether prestimulus activity is influenced by an individual's motivation to encode, and (2) at what point in time encoding-related activity emerges. Electrical brain activity was recorded while healthy male and female adults memorized series of words. Each word was preceded by a cue, which indicated the monetary reward that would be received if the following word was later remembered. Memory was tested after a short delay with a five-way recognition task to separate different sources of recognition. Electrical activity elicited by the reward cue predicted later memory of a word. Crucially, however, this was only observed when the incentive to memorize a word was high. Encoding-related activity preceded high-reward words that were later recollected. This activity started shortly after cue onset and persisted until word onset. Prestimulus activity thus not only signals cue-related processing but also an ensuing preparatory state. In contrast, reward-related activity was limited to the time period immediately after the reward cue. These findings indicate that engaging neural activity that benefits the encoding of an upcoming event is under voluntary control, reflecting a strategic preparatory state in anticipation of processing an event.

\section{Introduction}

It has recently become clear that anticipatory neural mechanisms play a role in the encoding of information into long-term memory. Using a variety of measures, studies have shown that neural activity elicited by a cue preceding an event can influence whether that event is later remembered (Adcock et al., 2006; Mackiewicz et al., 2006; Otten et al., 2006, 2010; Guderian et al., 2009; Park and Rugg, 2010). Encoding-related activity before an event ("prestimulus activity") occurs in addition to, but is dissociable from, encoding-related activity after an event ("poststimulus activity"). Thus, theories of memory formation need to take both into consideration.

In contrast to the large literature on poststimulus activity, little is known about prestimulus activity and encoding. Prestimulus activity can affect the encoding of several types of events (visual words, spoken words, scenes, neutral and emotional pictures), and functional magnetic resonance imaging (fMRI) studies have primarily implicated midbrain and medial temporal regions. An essential question is whether prestimulus activity is under voluntary control. One possibility is that neural activity can be engaged flexibly and used to strategically prepare for the encoding of an upcoming event (cf. Haynes et al., 2007). Alternatively, random variations in neural activity over time may be more or less conducive to effective encoding (cf. Meeter et al.,

Received Feb. 19, 2010; revised May 18, 2010; accepted June 9, 2010.

This work was supported by the Wellcome Trust. Stimulus presentation was programmed with the Cogent2000 software of the physics group of the Wellcome Trust Centre for Neuroimaging.

Correspondence should be addressed to Leun J. Otten, Institute of Cognitive Neuroscience, University College London, 17 Queen Square, London, WC1N 3AR, UK. E-mail: I.otten@ucl.ac.uk.

DOI:10.1523/JNEUROSCI.0915-10.2010

Copyright $\odot 2010$ the authors $\quad 0270-6474 / 10 / 309793-08 \$ 15.00 / 0$
2004), similar to what has been observed for other cognitive domains (Linkenkaer-Hansen et al., 2004; Hanslmayr et al., 2007; Hesselmann et al., 2008).

If anticipatory activity is under voluntary control, it would be expected to vary as a function of the degree to which an individual is motivated to encode. If there is a high incentive to encode information into memory, processes that benefit encoding should be maximally engaged, including any strategic preparation. To test this hypothesis, we used monetary rewards to motivate healthy adults to memorize series of words in a subsequent memory paradigm (Paller and Wagner, 2002). Each word was randomly preceded by one of two cues, which indicated whether a low or high reward would be received if that word was recognized in a later memory test (see Fig. 1). Memory was tested after a short delay with a five-way recognition judgment (Yonelinas et al., 2005; Woodruff et al., 2006; Voss and Paller, 2009) to assess what kind of memory processes are affected by prestimulus activity at encoding. Importantly, we measured electrical brain activity. Unlike fMRI (Adcock et al., 2006; Mackiewicz et al., 2006; Park and Rugg, 2010), EEG allows an assessment of how encoding-related processes evolve during the few hundred milliseconds leading up to an event. Prestimulus activity may reflect cue-related processing, the anticipation of an imminent event, or both. We assessed the role of voluntary control on anticipatory processes by contrasting electrical activity preceding low- and high-reward words that were later remembered versus forgotten. To address whether encoding-related activity is driven by reward-related activity (Adcock et al., 2006), we also compared encoding-related activity before an event with activity that differentiates the processing of low- and high-reward cues regardless of later memory performance. 


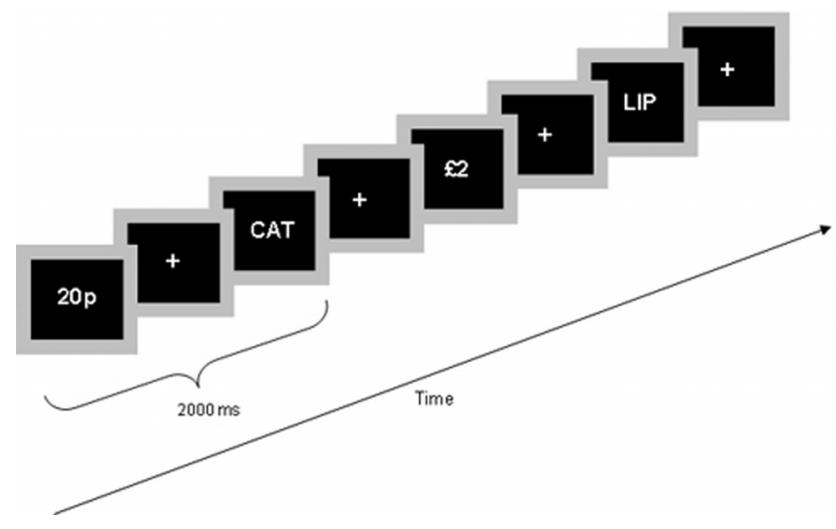

Figure 1. Example sequence of events during the study phase. Participants memorized series of words (e.g., "CAT" and "LIP"), each of which was preceded by the string "20p" written in black or the string " $£ 2$ " written in green. The string served as a prestimulus cue, indicating the amount of money that would be earned if the following word was correctly identified in a recognition memory test performed at $\sim 15 \mathrm{~min}$ after the study phase. The comparisons of interest involved electrical brain activity elicited in the $2000 \mathrm{~ms}$ cue-word interval.

\section{Materials and Methods}

Participants. The experimental procedures were approved by the University College London Research Ethics Committee. Twenty-four righthanded, native English speakers (mean age, 23 years; range of 19-33 years; nine men) volunteered to take part. All had normal or correctedto-normal vision and reported not to have a history of neurological or psychiatric illness. Color vision was checked with the abridged Ishihara plates. Each participant provided written informed consent before participating and was remunerated at a rate of $£ 7.50 / \mathrm{h}$ plus a monetary reward based on their memory performance (see below).

Procedure. The experiment consisted of an intentional encoding task, followed by a recognition memory test after a short delay. At study, participants viewed series of words, each of which was preceded by a cue (Fig. 1). The cue informed participants how much money they would earn if they recognized the following word during a later memory test. The low-reward cue consisted of the string "20p" written in black, and

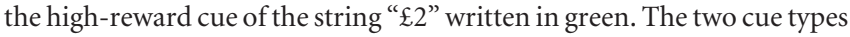
were equally likely to occur and randomly intermixed. Participants were told that at the end of the experiment they would be paid, in cash, $5 \%$ of the total amount of money they accrued during the memory test. On average, participants received $\mathfrak{E} 6.65$ (maximum possible amount of

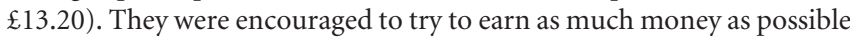
and use the cues to prepare themselves to memorize the upcoming words. To ensure that attention would be paid to all words, however, participants were also asked to make an alphabetic judgment on each word. They had to decide whether the first and last letters of a word were in alphabetical order and press one of two buttons as fast yet accurately as possible with the left or right index finger according to their decision. Responding hand was counterbalanced across participants. The study phase started with a short practice block (for which no monetary reward was received), followed by four blocks of 60 trials each. Brief rest periods were provided between blocks.

The recognition memory test started $\sim 15$ min after the end of the study phase. All studied words were presented again, intermixed with words not yet encountered in the experiment. Memory was tested with a five-way recognition judgment (Yonelinas et al., 2005) to focus the analyses on confidently recognized words and to better isolate recollection-based responses (Yonelinas, 2002). This approach has been used successfully in previous neuroimaging studies (Yonelinas et al., 2005; Woodruff et al., 2006; Voss and Paller, 2009). For each word, participants were instructed to press one of five buttons on a purpose-built response box that was designed to fit the right hand. If participants were confident that they had seen a word before and could recollect specific details about its initial occurrence (such as when the word was presented, what they thought about at the time, which decision they made about the word, or what it looked like on the screen), they were asked to press the button underneath their thumb. If they were confident that the word had been presented but could not retrieve any details, they should respond with their index finger. If they thought that the word had been presented but were not confident, a response was to be made with their middle finger. Finally, if they wanted to indicate that the word was new to the experiment, they were asked to press with their ring finger if they were not confident and with their little finger if they were. Participants were told that both speed and accuracy were important and that they should try to use the full range of buttons (cf. Yonelinas et al., 2005).

For each studied word that participants correctly recognized, their monetary reward would increase by either $£ 2$ or 20 p, depending on the type of cue that had preceded the word at study. For this purpose, all three "old" responses were counted as correct, regardless of the rating associated with the response. A $£ 2.50$ penalty was received for false alarms (new words falsely endorsed as old) to discourage participants from responding old all the time. No feedback was provided about the reward received on each trial. Instructions for the test phase were given in the delay period between study and test. A short practice block was given to familiarize participants with the memory test. They then performed six task blocks of 60 trials each, with short breaks in between.

Stimulus materials. The study and test sequences were constructed from a pool of 378 English words, taken from the study by Kučera and Francis (1967). Words were between four and eight letters in length and had a written frequency of 1-30 per million. Three sets of 120 words each were selected pseudorandomly from this pool, with the restriction that word lengths were approximately equal across sets and that half of the words in each set had first and last letters that were in alphabetical order. The sets were rotated across participants so that each word occurred equally often as a high-reward old item, a low-reward old item, or a new item. The words used for the low- and high-reward sets were randomly intermixed to form a study list of 240 items. A test list of 360 items was created by combining all studied words with the items from the remaining set. Both study and test lists were generated separately for each participant and split into blocks of 60 items each. The remaining words in the pool were used to create practice lists.

Stimuli were presented in central vision on a gray background. All words were shown in a black Helvetica font for $500 \mathrm{~ms}$. At study, cues were presented for $1 \mathrm{~s}$, starting $2 \mathrm{~s}$ before word onset. The remaining cue-word interval was filled with a fixation point (a plus sign). The fixation point was also presented between trials. At test, a neutral warning stimulus (a black exclamation mark) was shown $1 \mathrm{~s}$ before word onset. The time between successive cue onsets varied randomly between 6 and $7.5 \mathrm{~s}$ at study and between 4 and $5.5 \mathrm{~s}$ at test.

EEG acquisition. Electrical brain activity was recorded from 32 scalp sites using silver/silver-chloride electrodes embedded in an elastic cap according to an equidistant electrode montage (montage 10 at www. easycap.de/easycap/e/electrodes/13_M10.htm) and from two additional electrodes placed on the left and right mastoids. Vertical eye movements were recorded bipolarly from electrodes above and below the right eye and horizontal eye movements from electrodes at the outer canthi. The data were recorded relative to a midfrontal site. Impedances were kept below $5 \mathrm{k} \Omega$. Signals were amplified with a set of Contact Precision amplifiers, bandpass filtered between 0.01 and $35 \mathrm{~Hz}$ ( $3 \mathrm{~dB}$ roll off), and digitized (12-bit resolution) at a rate of $500 \mathrm{~Hz}$.

EEG analyses. Offline, the continuous EEG was digitally filtered between 0.05 and $20 \mathrm{~Hz}$ (96 dB roll off), downsampled to $100 \mathrm{~Hz}$, and algebraically re-referenced to averaged mastoids (reinstating the online reference site). Activity elicited by study cues and study words was analyzed separately to allow each to be aligned to the time period immediately preceding each event (cf. Otten et al., 2006, 2010). Epochs of 2560 ms duration surrounding cue and word onsets, including a $100 \mathrm{~ms}$ baseline, were extracted from the continuous record. Epochs were combined to create event-related potential (ERP) waveforms for each subject and electrode site, separately for study words in the low- and high-reward conditions later given "remember," "confident old," "nonconfident old," "nonconfident new," and "confident new" judgments. Epochs with drifts exceeding $\pm 50 \mu \mathrm{V}$, horizontal or non-blink eye movements, muscle artifacts, amplifier saturation, or incorrect alphabetic judgments were 
Table 1. Proportions of responses in the recognition memory task for studied and new words

\begin{tabular}{llll}
\hline & \multicolumn{3}{l}{ Word type } \\
\cline { 2 - 4 } Recognition judgment & Old, high reward & Old, low reward & New \\
\hline Remember & $0.41(0.20)$ & $0.12(0.12)$ & $0.01(0.01)$ \\
Confident old & $0.20(0.10)$ & $0.16(0.11)$ & $0.05(0.06)$ \\
Nonconfident old & $0.17(0.10)$ & $0.23(0.11)$ & $0.16(0.14)$ \\
Nonconfident new & $0.16(0.08)$ & $0.36(0.19)$ & $0.50(0.20)$ \\
Confident new & $0.07(0.09)$ & $0.13(0.12)$ & $0.28(0.20)$ \\
\hline
\end{tabular}

Values are across-subject means (SD). $n=24$.

excluded. A standard regression technique (Rugg et al., 1997) was used to minimize the contribution of blink artifacts to the waveforms.

The comparisons of interest contrasted ERP waveforms across lowand high-reward cues and across trials from which words were remembered or forgotten in the later recognition test (for details, see Results). Because memory was relatively good for high-reward items but poorer for low-reward items, comparisons were based on the maximum number of subjects who had at least 13 artifact-free trials in each relevant condition. This minimum inclusion threshold was based on previous investigations of encoding-related electrical brain activity (Otten et al., 2006, 2010; Kim et al., 2009). At most, three subjects contributed the minimum trial number for any comparison, with the remaining subjects contributing 16 trials or more. All 24 participants were included in the analyses of reward-related activity (mean trial numbers for high- and low-reward cues of 98 and 95, respectively). The analyses of encoding-related activity associated with later confident recognition were based on 19 participants in the high-reward condition (mean trial numbers of 57 and 25 for confidently recognized and forgotten words, respectively) and 15 participants in the low-reward condition (mean trial numbers of 35 and 37, respectively). Subsidiary analyses were performed in the high-reward condition to discern the role of recollection (Yonelinas et al., 2005). These analyses incorporated all 19 participants to isolate encodingrelated activity for later remember judgments and a subset of 14 participants to contrast that activity with encoding-related activity for later confident old judgments (the minimum mean number of trials was 23 in these analyses). The patterns of performance in the study and memory tasks did not differ across the sets of participants used for the ERP comparisons (see supplemental material, available at www.jneurosci.org).

Waveforms were quantified by measuring mean amplitude values in selected latency regions. The statistical reliability of amplitude and scalp distribution differences across conditions was evaluated with repeatedmeasures ANOVA, incorporating the Greenhouse-Geisser correction for violations of sphericity when appropriate (Keselman and Rogan, 1980). Scalp distribution differences were established after scaling the data to remove overall amplitude differences (McCarthy and Wood, 1985). The maximum/minimum scaling method was used rather than the vector length method to avoid the interpretational problems that can arise with the latter (Urbach and Kutas, 2002, 2006; Wilding, 2006). Unless stated otherwise, all 32 electrode sites were entered into the analyses.

\section{Results \\ Task performance}

Memory for words that were preceded by low- and high-reward cues at study is summarized in Table 1. Regardless of the type of recognition judgment, recognition accuracy (the proportion of hits minus the proportion of false alarms) was significantly higher for high- than low-reward words ( 0.56 vs 0.29 , pairedsample $\left.t_{(23)}=6.16, p<0.001\right)$. The superior memory performance for high-reward words was primarily attributable to a higher number of remember judgments. An ANOVA on the accuracy with which remember, confident old, and nonconfident old judgments were made revealed a significant interaction between reward and judgment type $\left(F_{(1.3,29.6)}=40.00, p<0.001\right)$. Bonferroni's corrected post hoc comparisons indicated that high- reward words received more remember and fewer nonconfident old judgments than low-reward words $\left(t_{(23)}=7.48\right.$ and -4.19 , respectively, both $p$ values $<0.001)$ but that low- and highreward words did not differ in the number of confident old judgments $(p=0.061)$. Importantly, although the number of remember judgments was smaller for low-reward words, memory discrimination in this category was well above chance ( $p_{\text {hit }}-$ $p_{\text {false alarm }}=0.11$, one-sample $\left.t_{23}=4.56, p<0.001\right)$. Recognition accuracy was greater than zero for all studied words except highreward words that were later nonconfidently recognized as old ( $p=0.712$; all other $p$ values $<0.001$ ).

At study, alphabetic judgments were made with approximately the same response speed regardless of whether a low- or high-reward cue preceded the word (mean reaction times of 1523 and $1551 \mathrm{~ms}$, respectively; $\left.t_{(23)}=-0.90, p=0.378\right)$. However, alphabetic judgments were somewhat more accurate after highreward cues ( 0.94 vs 0.92 correct, $\left.t_{(23)}=2.45, p=0.022\right)$. Anticipating the encoding-related ERP comparisons below, response times during the study phase did not differ significantly across low- and high-reward items that were later confidently recognized versus forgotten ( $p=0.611$ and 0.133 , respectively) or between high-reward items that were later recollected versus forgotten $(p=0.298)$.

\section{Reward-related activity}

Reward-related activity was investigated by contrasting ERP waveforms across low- and high-reward cues, regardless of later memory performance to the following words (Fig. 2). Cues signaling the potential of a high monetary reward gave rise to more positive-going ERPs between $\sim 200$ and $1100 \mathrm{~ms}$ after cue onset. This strongly resembles previous research (Otten et al., 1995; Yeung and Sanfey, 2004; Sato et al., 2005; Wu and Zhou, 2009). The sustained modulation observed here encompassed a larger positive (P) P2 deflection (Wastell and Kleinman, 1980; Lindholm and Koriath, 1985), a larger P3 deflection (Donchin and Coles, 1998), and finally a widespread deflection that persisted until just after $1 \mathrm{~s}$ after cue onset. These effects were quantified by measuring mean amplitude values in the 200-300 ms interval (surrounding the $\mathrm{P} 2$ in the group average), 300-600 ms interval (to capture the P3), and 600-1100 ms interval (to measure the late widespread effect). ANOVAs incorporating factors of reward and electrode site resulted in significant interactions for all three intervals $\left(F_{(2.8,64.1)}=17.06, p<0.001 ; F_{(3.6,82.6)}=10.67, p<\right.$ $0.001 ; F_{(3.8,87.3)}=4.75, p=0.002$, respectively). Direct comparisons across time confirmed three qualitatively distinct rewardrelated activities. Significant interactions between interval, reward, and scalp site emerged when comparing each of the three time windows with each other (all $p$ values $<0.001$ ). These interactions remained significant after scaling the data (McCarthy and Wood, 1985), indicating reliable scalp distribution differences across time. The amplitude of the attention-sensitive P1 and negative (N) N1 deflections did not differ across low- and high-reward cues (see supplemental material, available at www.jneurosci.org).

\section{Encoding-related activity preceding words}

Neural activity related to successful encoding was identified with the subsequent memory approach (Sanquist et al., 1980). Subsequent memory effects (study activity that differs as a function of subsequent memory performance) were assessed in each reward condition. To that end, ERP waveforms elicited by low- and highreward cues were contrasted depending on whether the following word was remembered or forgotten in the later recognition test. The initial analyses focused on neural activity associated with 
confident recognition (analyses on all recognized words gave rise to the same, but smaller, effects). Figure 3 shows the group-averaged waveforms elicited by cues preceding words that were later forgotten (i.e., given new judgments) or confidently recognized (i.e., given remember or confident old judgments). Words in the high-reward condition display a pronounced subsequent memory effect before word onset. Relative to forgotten words, confidently recognized words are preceded by a more positive-going ERP waveform from $\sim 300 \mathrm{~ms}$ after cue onset until the end of the cue-word interval, becoming smaller over time. The effect has a widespread scalp distribution. In stark contrast, ERP waveforms elicited by cues in the low-reward condition virtually overlap.

Statistical analyses confirmed these observations. The sustained prestimulus subsequent memory effect in the highreward condition was quantified in successive time intervals to evaluate possible differences in amplitude or scalp distribution over time. The same intervals as used for the reward-related analyses were used so that encoding-related activity could be contrasted with reward-related activity. Encoding-related activity was also quantified in an additional interval at the end of the cue-word interval, because an encoding, but not reward, effect is apparent in this interval. The 1100$2000 \mathrm{~ms}$ interval was used for this late effect (identical findings resulted with a temporally more restricted interval just before word onset). ANOVAs demonstrated that subsequent memory performance significantly modulated the ERPs elicited by highreward cues in the 600-1100 ms interval (main effect of subsequent memory, $F_{(1,18)}=5.96, p=0.025$; all other $p$ values $>0.072$ ). For low-reward cues, no significant subsequent memory effect emerged in any interval ( $p$ values $>0.227$ ).

A potential problem with the above analyses relates to the unequal trial numbers contributing to the ERP waveforms for remembered and forgotten words. In the low-reward condition, approximately equal numbers of trials were available for the subsequent memory comparison. In the high-reward condition, however, twice as many words were later confidently recognized than forgotten. It has been demonstrated that unequal trial numbers can affect differences between ERP waveforms (Thomas et al., 2004; Luck, 2004, 2005). The mean area measure we use to quantify ERPs is relatively robust against variations in trial numbers (Luck, 2004; Thomas et al., 2004). Nonetheless, we performed a bootstrap analysis (Efron, 1979) to ensure that the subsequent memory effect observed in the high-reward condition cannot be accounted for by unequal trial numbers.

The bootstrap procedure was similar to that reported by Mathewson et al. (2009). For each reward condition, we first determined the minimum number of trials available for the subsequent memory comparison in each subject. This number of trials was selected at random from all words that the subject later confidently recognized, returning a trial to the pool after each selection (Efron, 1979). The same was done for the forgotten
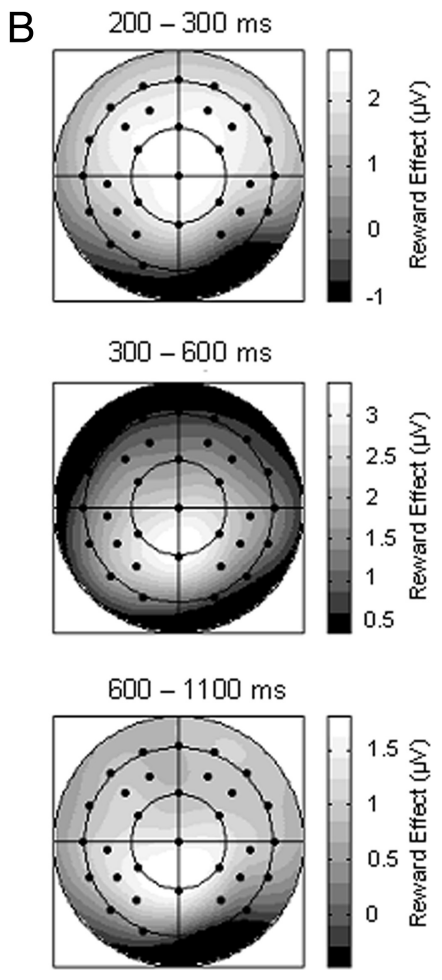
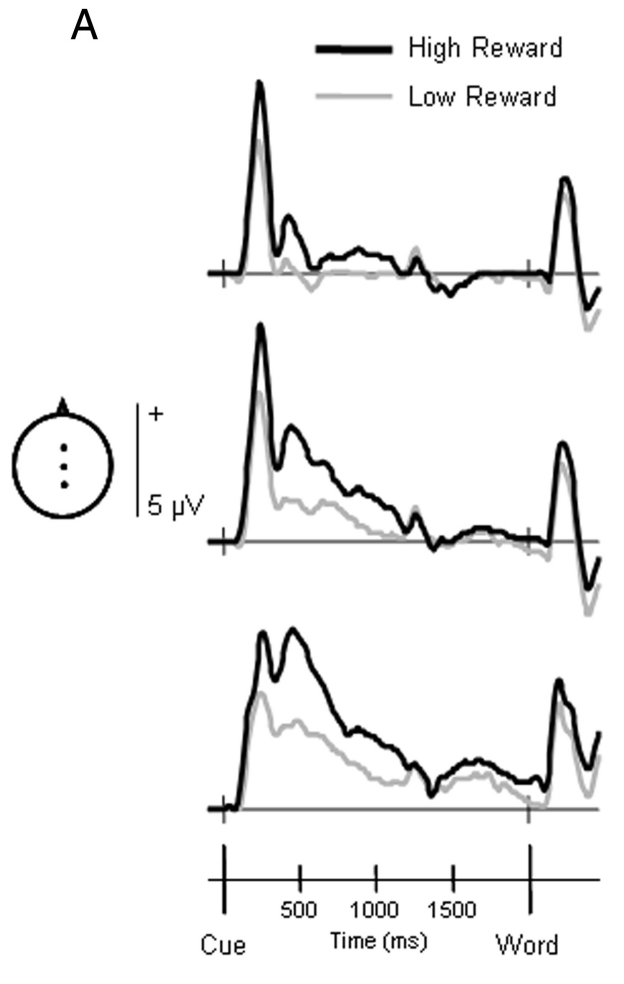
.

Figure 2. Reward-related neural activity. A, Group-averaged ERP waveforms elicited by low-and high-reward cues, regardless of memory performance to the following words. Waveforms are shown for three representative midline electrode sites (equivalent , $(z$, and Pz of the international 10/10 system). Positive values are plotted upward. For graphical purposes, the waveform scalp distribution of reward-related activity (difference between high- and low-reward cues) in the 200-300, 300-600, and $600-1100$ ms latency regions after cue onset. Maps are range scaled.

word category. Group-averaged ERPs were then formed from the individual subjects' waveforms. Finally, mean amplitude values were measured in the group-averaged ERPs in the 600-1100 ms interval across all electrode sites for confidently recognized words, forgotten words, and the difference between the two. This process was iterated 10,000 times. Figure 4 shows the resulting distributions of sample means for low- and high-reward words that were later confidently recognized or forgotten. A clear separation is apparent between words in the high-reward, but not low-reward, condition. Of the 10,000 samples in the high-reward condition, 9945 showed mean amplitude values that were larger for remembered than forgotten words. The $95 \%$ confidence interval in this condition indicated a subsequent memory effect that lies between 0.25 and $1.77 \mu \mathrm{V}$. In the low-reward condition, 3163 samples showed amplitude values that were larger for remembered words. The $95 \%$ confidence interval in this condition indicated a subsequent memory effect that included zero and hence no significant difference. These bootstrap analyses thus corroborate that prestimulus activity affects encoding in the high-reward condition even when equal trial numbers are used for the subsequent memory comparisons.

Next, the analyses were restricted to high-reward words that were later given remember judgments to isolate encoding-related activity leading to later recollection. These words showed the same widespread, positive-going subsequent memory effect before word onset as observed across all confidently recognized words. However, the effect was more pronounced and extended until word onset. Figure 5 illustrates recollectionrelated encoding activity (note that the waveforms in Fig. 5A are based on the subset of participants described in the next 


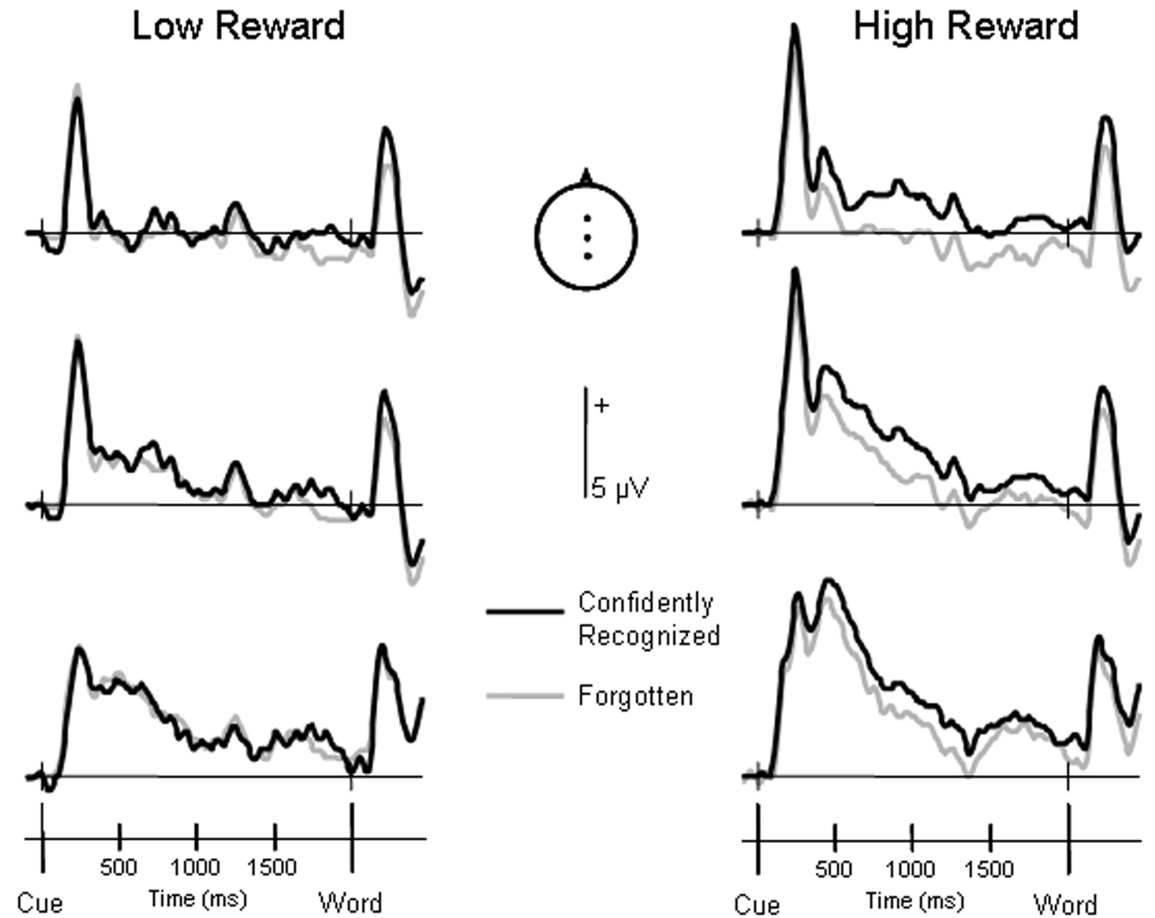

Figure 3. Encoding-related neural activity before word onset predicting later confident recognition. Group-averaged ERP waveforms elicited by low- and high-reward cues at three midline electrode sites (equivalent to $\mathrm{Fz}, \mathrm{Cz}$, and $\mathrm{Pz}$ of the international 10/10 system), separated as a function of later memory performance to the following words. Positive values are plotted upward. On the left are waveforms elicited by low-reward cues, overlaid according to whether the following word was later confidently recognized (given a remember or confident old judgment) or forgotten (judged as new). No differences are apparent. On the right are waveforms elicited by high-reward cues, again contrasting later confidently recognized and forgotten words. A positive-going subsequent memory effect emerges from $\sim 300 \mathrm{~ms}$ after cue onset.
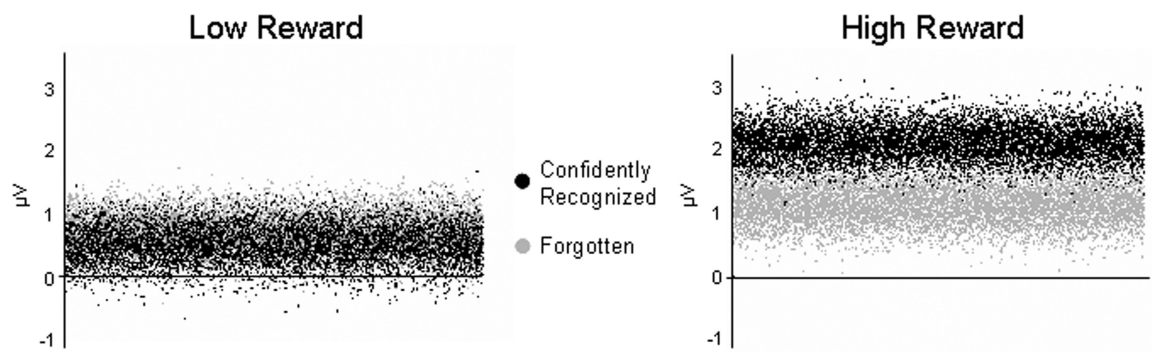

Figure 4. Results of a bootstrap analysis on encoding-related neural activity before word onset in the low- and high-reward conditions. In each condition, equal numbers of confidently recognized and forgotten words were selected at random with replacement for each subject. The group average was then computed, and the mean amplitude in the $600-1100$ ms interval was measured across all 32 electrode sites. This process was repeated 10,000 times. The resulting sampling distributions are shown here. Each gray dot represents a sample value for forgotten words, and each black dot represents a sample value for recognized words. Bootstrap iterations are displayed along the horizontal axes. Distributions for the low-reward condition are shown on the left, and those for the high-reward condition are shown on the right. There is a clear separation between the distributions of recognized and forgotten words in the high-reward condition. No such separation is apparent in the low-reward condition. For statistical comparisons, see Results.

paragraph; recollection-related activity was identical in both groups). ANOVAs on the mean amplitudes in the four time intervals after cue onset showed significant effects of subsequent memory performance from $300 \mathrm{~ms}$ onward when comparing recollected and forgotten words (main effects of subsequent memory in the 300-600, 600-1100, and 1100-2000 ms intervals, $F_{(1,18)}=8.89, p=0.008 ; F_{(1,18)}=9.72, p=0.006 ; F_{(1,18)}=6.60$, $p=0.019$, respectively; and an interaction between subsequent memory and electrode site in the 300-600 $\mathrm{ms}$ interval, $F_{(3.7,65.8)}=$ $2.59, p=0.050$; all other $p$ values $>0.079)$. Contrasting the effects across the three time intervals spanning 300-2000 ms did not reveal significant differences (all $p$ values $>0.627)$. A bootstrap analysis on the mean values in the 300-2000 ms interval indicated a significant subsequent memory effect even when trial numbers were equated across recollected and forgotten items (9992 of the 10,000 iterations showing a positive effect).

A direct comparison between highreward words that were later given remember, confident old, or new judgments in the subset of 14 participants who had at least 13 artifact-free trials in each category confirmed that the prestimulus subsequent memory effect is specific to recollected words. As shown in Figure $5 A$, contrary to the clear subsequent memory effect preceding later recollected words, no differences emerged before the onset of words later judged as confident old or new. An ANOVA evaluating prestimulus activity between the latter two classes of word did indeed not reveal significant effects $(p$ values $>0.363$, apart from $p=$ 0.070 for the interaction between electrode site and subsequent memory in the 200-300 ms interval). In contrast, the comparison between activity preceding later recollected and confident old words gave rise to significant differences from $200 \mathrm{~ms}$ onward (interaction between subsequent memory and electrode site in the $200-300$ and $300-600 \mathrm{~ms}$ intervals, $p$ values $<0.034$; significant main effects from 300 ms onward, $p$ values $<0.031$; all other $p$ values $>0.051)$. The effect between recollected and forgotten words was the same as described for the larger group in the previous paragraph.

The data thus indicate that electrical brain activity before word onset influences the effectiveness with which a word is encoded but only when there is a high monetary incentive to remember the word later. This is especially evident before words that are later recollected. An analysis of the attention-sensitive P1 and N1 elicited by high-reward words did not suggest that the effect in this condition can be explained by attentionrelated processes (cf. Otten et al., 2006, 2010) (see supplemental material, available at www.jneurosci.org). The absence of a prestimulus subsequent memory effect for words in the low-reward condition cannot be explained by the relatively poor memory performance in that condition. An analysis in which the number of remembered low-reward words was increased by including nonconfidently recognized words (accuracy of which was above chance) did not bring out any effect ( $p$ values $>0.385$ ). Likewise, restricting the analyses to words that were later recollected, and for which memory was consequently good, did also not provide any hint of a prestimulus subsequent memory effect in the face of low monetary remuneration ( $p$ values $>0.213$ ). 
Finally, we contrasted prestimulus subsequent memory effects across the low- and high-reward conditions, although there were relatively few participants who had at least 13 remembered and forgotten items in both conditions $(n=13)$. We compared the high-reward condition in which the prestimulus effect was most evident (recollected vs forgotten words) with the low-reward condition in which memory was well above chance (confidently recognized vs forgotten words). Activity was collapsed across the 300-2000 ms interval because the effect occurred throughout this period. An ANOVA on the mean amplitudes for remembered and forgotten low- and highreward words did not give rise to a significant interaction between subsequent memory and reward $\left(F_{(1,12)}=1.21, p=\right.$ $0.295)$. However, a supplementary analysis using the bootstrap procedure described previously did signal a significant difference between reward conditions. In this analysis, subsequent memory effects were compared between low- and high-reward words, keeping the maximum number of trials available for each condition to increase statistical sensitivity. The resulting distribution of sampling means showed that, of 10,000 iterations, 9824 contained effects that were larger in the high-reward condition. The associated 95\% confidence interval of the difference between reward conditions ranged between 0.07 and $2.26 \mu \mathrm{V}$. In this sampling distribution, the subsequent memory effect for low-reward words did not differ from zero (95\% confidence limits of -0.62 and $0.84 \mu \mathrm{V}$ ), whereas that for high-reward words did (95\% confidence limits of 0.50 and $2.10 \mu \mathrm{V}$ ). These analyses strengthen the conclusion that prestimulus activity only influences encoding in the face of a high monetary reward.

\section{Encoding-versus reward-related activity}

It has been suggested that similar neural bases underlie memory encoding and reward (Adcock et al., 2006). To address this issue, we compared reward-related activity with encoding-related activity before an event leading to later recollection. As explained above, reward as well as encoding modulated activity until 1100 ms after cue onset. In addition, encoding affected activity in the later 1100-2000 ms interval. The differences in time of occurrence suggest a temporal separation between reward- and encoding-related activity. Perhaps more importantly, a direct comparison between encoding- and reward-related activity in the three time intervals up to $1100 \mathrm{~ms}$ revealed a significant interaction between effect type and electrode site for the 200-300 and 300-600 ms intervals $\left(F_{(3.3,59.2)}=2.85, p=0.040\right.$ and $F_{(3.7,67.5)}=4.18, p=0.005$, respectively; all other $p$ values $>0.291)$. The latter remained significant after scaling. This points to a qualitative difference between these two types of activity in at least the later time window. Whereas the reward effect was maximal over posterior scalp sites between 300 and $600 \mathrm{~ms}$, the encoding

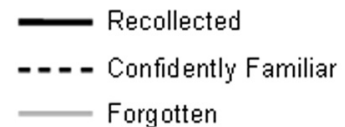

B
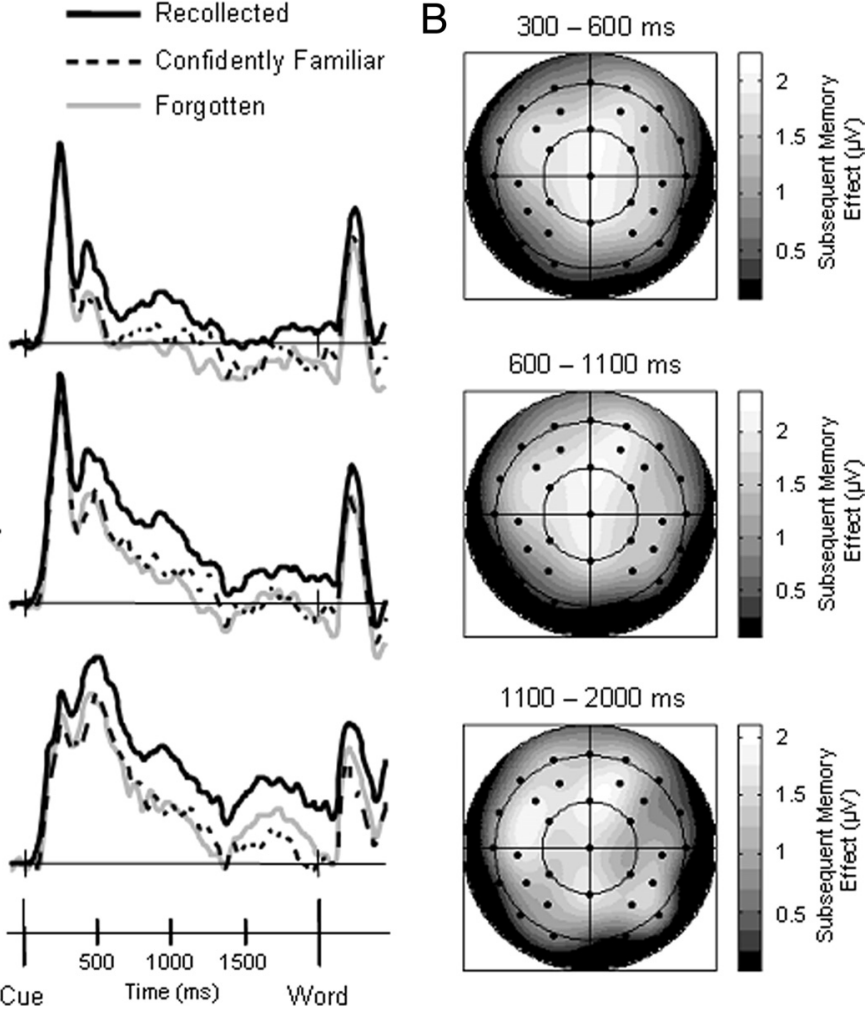

$1100-2000 \mathrm{~ms}$

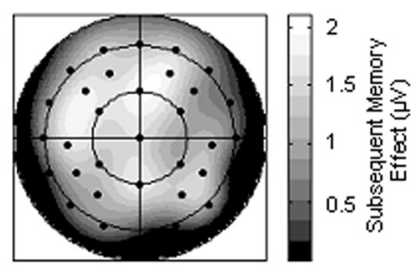

Figure 5. Encoding-related neural activity before word onset predicting later recollection in the high-reward condition. $\boldsymbol{A}$, Group-averaged ERP waveforms elicited by high-reward cues at the three midline electrode sites (equivalent to Fz, Cz, and Pz of the international 10/10 system). Waveforms are overlaid according to whether the following word was recollected (given a remember (hive subse-

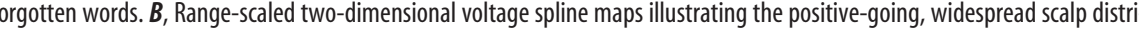
Note that these maps were computed across all participants with sufficient numbers of recollected and forgotten trials to maximize the signal-to-noise ratio, not just the subset shown in $\boldsymbol{A}$.

effect was spatially widespread. Thus, reward and encoding activity are dissociable in their time of occurrence and, at least sometimes, their neural generators.

\section{Discussion}

We examined whether prestimulus activity that benefits memory encoding is under voluntary control. Three main findings emerged. First, electrical brain activity elicited by a cue presented before a word predicted later memory of the word but only when the cue indicated a high monetary reward for remembering the word. This encoding-related activity started shortly after cue onset and persisted until the word appeared. Second, encodingrelated activity was most pronounced before words that were later recollected. Third, encoding-related activity differed, at least in part, from reward-related activity.

These findings provide strong evidence that some anticipatory processes that benefit encoding are under voluntary control. The observation that prestimulus activity affects encoding in a highreward, but not low-reward, condition implies that individuals are in command of the use of such activity. High- and low-reward trials were randomly intermixed. If prestimulus influences on encoding are the result of random fluctuations of neural activity over time, subsequent memory effects should have been evident on both types of trial. The lack of an effect in the low-reward 
condition cannot be explained by the poorer memory in that condition (no effect was observed for low-reward words that were confidently remembered or recollected) or by individuals simply ignoring words on these trials (each word required an alphabetic decision, ensuring at least a minimum level of attention). Instead, it must be possible to engage encoding-related activity strategically depending on the need for such activity to help encode a forthcoming event. In the present case, this activity is engaged when a monetary reward increases the incentive to learn. The higher the motivation is to learn, the more likely it is that processes that aid encoding will be used, including those before an event.

The conclusion that encoding-related prestimulus activity is under voluntary control raises the interesting possibility that some memory deficits arise because an individual did not prepare appropriately before encountering an event. All else equal, this would be expected to result in an impoverished representation of the event in memory, which will be more difficult to retrieve later (Craik and Lockhart, 1972). By the same token, it may be possible to improve memory by optimizing the probability that encoding-related activity before an event is engaged. This may be accomplished with rewards, such as in the present case, or by teaching individuals to prepare themselves in such a way that benefits encoding.

Prestimulus activity did not affect all types of memory judgments. Events may be recognized because they bring back contextual details from when they were first encountered, because they engender a sense of familiarity, or both (Tulving, 1985, Yonelinas, 2002; Wixted, 2007). With one exception (Otten et al., 2010), previous studies have not been able to address what kind of memory judgment is affected by prestimulus activity at encoding. These studies either focused on one kind of memory judgment or collapsed across judgments (Adcock et al., 2006; Mackiewicz et al., 2006; Otten et al., 2006; Guderian et al., 2009; Park and Rugg, 2010). Here, prestimulus influences on encoding were most pronounced for high-reward items later given remember judgments. This suggests that prestimulus activity is especially important for encoding processes that lead to later recollection (i.e., episodic memory). Although there is no perfect way to measure recollection, we used a five-way recognition judgment that does not confound remember judgments with high confidence (Yonelinas et al., 2005). It thus seems that prestimulus activity is especially important for the creation of memory representations that are contextually rich (Tulving, 1983). Alternatively, remember judgments may have been made for items with especially strong representations (Wais et al., 2008). In this respect, it will be interesting to determine how prestimulus activity affects encoding in individuals who experience difficulty with episodic memory processes, such as Alzheimer's patients and the elderly (for review, see Hedden and Gabrieli, 2004).

The observation that neural activity before an event can influence memory formation is consistent with recent fMRI studies, especially that of Adcock et al. (2006) (see also Mackiewicz et al., 2006; Park and Rugg, 2010). In contrast to these studies, however, the present results pinpoint anticipatory processes to neural activity just before an event. The relatively sluggish hemodynamic response does not allow a determination of the exact time at which prestimulus activity occurs. Activity identified in fMRI studies may therefore reflect the processing of the prestimulus cue, an ensuing preparatory state, or both. Interestingly, we did not observe a significant subsequent memory effect in the few hundred milliseconds before word onset when the analyses were confined to confidently recognized words (cf. Adcock et al., 2006). For words that were later recollected, encoding-related activ- ity started at $\sim 200 \mathrm{~ms}$ after the presentation of the cue and persisted until word onset. The neural and cognitive processes that benefit encoding thus took effect when the relevant aspects of the cue had been processed and were maintained throughout the cue-word interval. An interesting question is whether anticipatory influences on encoding are specific to situations in which explicit prestimulus cues are used or whether any situation in which an upcoming event can be predicted benefits from a preparatory state.

Strikingly, the type of electrical brain activity that predicted later recollection in the present study differed from that observed previously. Otten et al. $(2006,2010)$ demonstrated that a negative ERP modulation over frontal scalp sites preceded the successful encoding of visual and auditory events. Here, a spatially widespread positive modulation was associated with effective memory formation. The polarity and scalp distribution differences indicate that qualitatively different types of neural activity support encoding in each case (Rugg and Coles, 1995). There is thus not one but at least two types of prestimulus activity that benefit encoding. This means that there are multiple ways to prepare to encode an upcoming event. It will be crucial to understand what type of prestimulus activity is engaged on any occasion and how each contributes to effective encoding.

The negative ERP modulation observed in previous incidental encoding studies may index the degree to which semantic processing resources are prepared ahead of stimulus presentation in aid of an upcoming semantic decision (Otten et al., 2006, 2010). This is because the effect has thus far only been observed in semantically oriented encoding tasks (animacy or imagery judgments). The same interpretation for the positive deflection is unlikely given the qualitative differences in underlying neural activity. One possibility is that this deflection reflects another type of semantic preparation. Encoding was intentional rather than incidental in the current study, which involves more elaborate semantic and associative processes (Fabiani et al., 1990). Another possibility is that the deflection signals an influence of attention on high-reward trials. Individuals are likely to be generally more prepared on some trials than others, and it is well known that attention affects encoding success (Craik et al., 1996). The data do not provide strong support for this idea, but we cannot entirely rule out at least a partial role of attention. Alphabetic judgments were indeed somewhat more accurate on highreward trials. However, the speed with which decisions were made at study did not differ according to later memory performance or reward condition. Attention-sensitive ERP deflections elicited by cues and words also did not vary.

Instead, the positive ERP modulation may reflect rewardrelated processes. Reward-related activity was engaged between 200 and $1100 \mathrm{~ms}$ after cue onset and encompassed three effects. The initial two likely reflect the ease with which perceptual information conveyed by a high-reward cue is processed (Lindholm and Koriath, 1985) and the consequential updating of the internal environment (Donchin, 1981; Otten et al., 1995; Yeung and Sanfey, 2004). Perhaps not by coincidence, the final rewardrelated effect had a similar widespread scalp distribution as the encoding-related effect in the same time interval. This may suggest an interplay between reward and encoding in service of an upcoming event (cf. Adcock et al., 2006; Wittmann et al., 2007). A reward-related process may be kept in working memory, giving rise to a preparatory state that affects the success with which information is encoded into long-term memory.

More work will be needed to fully understand the functional role of prestimulus activity in memory formation. Regardless, the current experiment allows three firm conclusions. First, the pre- 
stimulus activity observed here is under an individual's control. Second, this activity reflects a preparatory state because it lasts until the onset of an event. Third, preparatory activity affects only some memory judgments. By comparing the activity observed in the present study with that in others (Otten et al., 2006, 2010), it is clear that there exists more than one kind of prestimulus activity. Thus, there are multiple ways to prepare to encode an upcoming event. Anticipatory neural activity is known to affect other cognitive domains as well, such as attention (Driver and Frith, 2000), problem solving (Kounios et al., 2006), perception (Mathewson et al., 2009), and memory inhibition (Hanslmayr et al., 2009). It will be of considerable interest to determine whether, and how, these different types of prestimulus activity relate to one another.

\section{References}

Adcock RA, Thangavel A, Whitfield-Gabrieli S, Knutson B, Gabrieli JD (2006) Reward-motivated learning: mesolimbic activation precedes memory formation. Neuron 50:507-517.

Craik FI, Lockhart RS (1972) Levels of processing: a framework for memory research. J Verb Learn Verb Behav 11:671-684.

Craik FI, Govoni R, Naveh-Benjamin M, Anderson ND (1996) The effects of divided attention on encoding and retrieval processes in human memory. J Exp Psychol Gen 125:159-180.

Donchin E (1981) Surprise! Surprise? Psychophysiology 18:493-513.

Donchin E, Coles MGH (1998) Context updating and the P300. Behav Brain Sci 21:152-154.

Driver J, Frith C (2000) Shifting baselines in attention research. Nat Rev Neurosci 1:147-148.

Efron B (1979) Bootstrap methods: another look at the jackknife. Ann Statistics 7:1-26.

Fabiani M, Karis D, Donchin E (1990) Effects of mnemonic strategy manipulation in a Von Restorff paradigm. Electroencephalogr Clin Neurophysiol 75:22-35.

Guderian S, Schott BH, Richardson-Klavehn A, Düzel E (2009) Medial temporal theta state before an event predicts episodic encoding success in humans. Proc Natl Acad Sci U S A 106:5365-5370.

Hanslmayr S, Aslan A, Staudigl T, Klimesch W, Herrmann CS, Bäuml KH (2007) Prestimulus oscillations predict visual perception performance between and within subjects. Neuroimage 37:1465-1473.

Hanslmayr S, Leipold P, Pastötter B, Bäuml KH (2009) Anticipatory signatures of voluntary memory suppression. J Neurosci 29:2742-2747.

Haynes JD, Sakai K, Rees G, Gilbert S, Frith C, Passingham RE (2007) Reading hidden intentions in the human brain. Curr Biol 17:323-328.

Hedden T, Gabrieli JD (2004) Insights into the ageing mind: a view from cognitive neuroscience. Nat Rev Neurosci 5:87-96.

Hesselmann G, Kell CA, Eger E, Kleinschmidt A (2008) Spontaneous local variations in ongoing neural activity bias perceptual decisions. Proc Natl Acad Sci U S A 105:10984-10989.

Keselman HJ, Rogan JC (1980) Repeated measures F tests and psychophysiological research: controlling the number of false positives. Psychophysiology 17:499-503.

Kim AS, Vallesi A, Picton TW, Tulving E (2009) Cognitive association formation in episodic memory: evidence from event-related potentials. Neuropsychologia 47:3162-3173.

Kounios J, Frymiare JL, Bowden EM, Fleck JI, Subramaniam K, Parrish TB, Jung-Beeman M (2006) The prepared mind: neural activity prior to problem presentation predicts subsequent solution by sudden insight. Psychol Sci 17:882-890.

Kučera H, Francis WN (1967) Computational analysis of present-day American English. Providence, RI: Brown.

Lindholm E, Koriath JJ (1985) Analysis of multiple event-related potential components in a tone discrimination task. Int J Psychophysiol 3:121-129.

Linkenkaer-Hansen K, Nikulin VV, Palva S, Ilmoniemi RJ, Palva JM (2004) Prestimulus oscillations enhance psychophysical performance in humans. J Neurosci 24:10186-10190.

Luck SJ (2004) Ten simple rules for designing ERP experiments. In: Eventrelated potentials: a methods handbook (Handy TC, ed). Cambridge, MA: Massachusetts Institute of Technology.

Luck SJ (2005) An introduction to the event-related potential technique. Cambridge, MA: Massachusetts Institute of Technology.
Mackiewicz KL, Sarinopoulos I, Cleven KL, Nitschke JB (2006) The effect of anticipation and the specificity of sex differences for amygdala and hippocampus function in emotional memory. Proc Natl Acad Sci USA 103:14200-14205.

Mathewson KE, Gratton G, Fabiani M, Beck DM, Ro T (2009) To see or not to see: prestimulus alpha phase predicts visual awareness. J Neurosci 29:2725-2732.

McCarthy G, Wood CC (1985) Scalp distributions of event-related potentials: an ambiguity associated with analysis of variance models. Electroencephalogr Clin Neurophysiol 62:203-208.

Meeter M, Murre JM, Talamini LM (2004) Mode shifting between storage and recall based on novelty detection in oscillating hippocampal circuits. Hippocampus 14:722-741.

Otten LJ, Gaillard AW, Wientjes CJ (1995) The relation between eventrelated brain potential, heart rate, and blood pressure responses in an S1-S2 paradigm. Biol Psychol 39:81-102.

Otten LJ, Quayle AH, Akram S, Ditewig TA, Rugg MD (2006) Brain activity before an event predicts later recollection. Nat Neurosci 9:489-491.

Otten LJ, Quayle AH, Puvaneswaran B (2010) Prestimulus subsequent memory effects for auditory and visual events. J Cogn Neurosci 22:1212-1223.

Paller KA, Wagner AD (2002) Observing the transformation of experience into memory. Trends Cogn Sci 6:93-102.

Park H, Rugg MD (2010) Prestimulus hippocampal activity predicts later recollection. Hippocampus 20:24-28.

Rugg MD, Coles MGH (1995) The ERP and cognitive psychology: conceptual issues. In: Electrophysiology of mind (Rugg MD, Coles MGH, eds). Oxford: Oxford UP.

Rugg MD, Mark RE, Gilchrist J, Roberts RC (1997) ERP repetition effects in indirect and direct tasks: effects of age and interitem lag. Psychophysiology 34:572-586.

Sanquist TF, Rohrbaugh JW, Syndulko K, Lindsley DB (1980) Electrocortical signs of levels of processing: perceptual analysis and recognition memory. Psychophysiology 17:568-576.

Sato A, Yasuda A, Ohira H, Miyawaki K, Nishikawa M, Kumano H, Kuboki T (2005) Effects of value and reward magnitude on feedback negativity and P300. Neuroreport 16:407-411.

Thomas DG, Grice JW, Najm-Briscoe RG, Miller JW (2004) The influence of unequal numbers of trials on comparisons of average event-related potentials. Dev Neuropsychol 26:753-774.

Tulving E (1983) Elements of episodic memory. Oxford: Clarendon.

Tulving E (1985) Memory and consciousness. Can Psychol 26:1-12.

Urbach TP, Kutas M (2002) The intractability of scaling scalp distributions to infer neuroelectric sources. Psychophysiology 39:791-808.

Urbach TP, Kutas M (2006) Interpreting event-related brain potential (ERP) distributions: implications of baseline potentials and variability with application to amplitude normalization by vector scaling. Biol Psychol 72:333-343.

Voss JL, Paller KA (2009) Remembering and knowing: electrophysiological distinctions at encoding but not retrieval. Neuroimage 46:280-289.

Wais PE, Mickes L, Wixted JT (2008) Remember/know judgments probe degrees of recollection. J Cogn Neurosci 20:400-405.

Wastell DG, Kleinman D (1980) Evoked potential correlates of visual selective attention. Acta Psychol (Amst) 46:129-140.

Wilding EL (2006) The practice of rescaling scalp-recorded event-related potentials. Biol Psychol 72:325-332.

Wittmann BC, Bunzeck N, Dolan RJ, Düzel E (2007) Anticipation of novelty recruits reward system and hippocampus while promoting recollection. Neuroimage 38:194-202.

Wixted JT (2007) Dual-process theory and signal-detection theory of recognition memory. Psychol Rev 114:152-176.

Woodruff CC, Hayama HR, Rugg MD (2006) Electrophysiological dissociation of the neural correlates of recollection and familiarity. Brain Res 1100:125-135.

Wu Y, Zhou X (2009) The P300 and reward valence, magnitude, and expectancy in outcome evaluation. Brain Res 1286:114-122.

Yeung N, Sanfey AG (2004) Independent coding of reward magnitude and valence in the human brain. J Neurosci 24:6258-6264.

Yonelinas AP (2002) The nature of recollection and familiarity: a review of 30 years of research. J Mem Lang 46:441-517.

Yonelinas AP, Otten LJ, Shaw KN, Rugg MD (2005) Separating the brain regions involved in recollection and familiarity in recognition memory. J Neurosci 25:3002-3008. 